\title{
Conflict Alerts for Aircraft Conducting Visual Approaches
}

\author{
Huabin Tang ${ }^{1}$ \\ NASA Ames Research Center, Moffett Field, CA 94035
}

\begin{abstract}
It is common for aircraft to conduct visual and instrument final approaches to a single runway or multiple parallel runways. Useful, nonexcessive safety alerts on aircraft conducting visual approaches are helpful to air traffic controllers, though pilots are responsible for separation with the preceding aircraft. A variety of visual approaches to various runway configurations are studied, and a set of safety alert thresholds is proposed. Fast-time simulations with recorded real-world air traffic data of mostly visual approach flights are performed on a prototype tactical separation assurance system for terminal airspace. Alerts are generated -- with both the standard separation thresholds and the proposed safety alert thresholds -- and compared with those from the Conflict Alert (CA) functionality in the Standard Terminal Automation Replacement System (STARS). The results show that the number of Mode-C Intruder alerts generated was reduced $76 \%$ as compared to STARS CA. The nuisance alerts generated by assuming visual to be instrument approaches was reduced by $92 \%$ when the proposed safety alert thresholds were used and visual approaches were assumed.
\end{abstract}

\section{Introduction}

$\mathrm{T}$ oday air traffic controllers are responsible for separation of air traffic, utilizing decision support tools at their disposal. Both the CARTS (Common Automated Radar Terminal System) ${ }^{1}$ and the newer STARS (Standard Terminal Automation Replacement System $)^{2}$ have a Conflict Alert (CA) functionality that alerts the air traffic controller when two aircraft get dangerously close in the US terminal airspace. The dangerous proximity ${ }^{1,2}$ refers approximately to a horizontal separation of $1 \mathrm{NM}$ and a vertical separation of $300 \mathrm{ft}$. However, losses of standard separation, which is typically a horizontal separation of $3 \mathrm{NM}$ and a vertical separation of $1000 \mathrm{ft}$ in terminal airspace with various other criteria such as wake turbulence as defined in FAA Order JO $7110.65 \mathrm{~V},{ }^{3}$ have been a significant safety concern, for which the CA functionality was not designed. An automated alerting function based on standard separation would spare air traffic controllers unnecessary and imprecise manual estimations of losses of separation and thus ensure safer terminal operations.

The inherent complexities of terminal airspace operations pose a number of challenges in the development of tools that automatically alert the air traffic controller to potential separation conflicts. Routine large-angle turns before final approaches requires proper handling to prevent a proliferation of nuisance alerts. Spacing aircraft near standard separation thresholds to maximize arrival and departure throughput increases the difficulty of predicting separation conflicts without causing too many false alerts. The difficulty also stems from the dynamic and complex nature of the standard separation criteria, which depend on relative course heading, weight classes, locations along the localizer, and other factors. ${ }^{3}$ Further challenge, as explained in the next section, comes from the need to provide safety alerts for potential conflicts involving aircraft conducting visual approaches to a single runway or multiple parallel runways simultaneously.

Recently a prototype system called the Terminal Tactical Separation-Assured Flight Environment (T-TSAFE) has been developed ${ }^{4}$. T-TSAFE takes into account available flight intent information for predicting aircraft trajectories and employs a single-trajectory algorithm for conflict detection. T-TSAFE alerts the controller to predicted losses of separation based on the standard separation criteria with a look-ahead time of about two minutes. Analysis shows that T-TSAFE has an average alert lead time of $\sim 30$ seconds (to first loss of separation) and a false alert rate of $\sim 10 \%$ of the total alerts. ${ }^{5}$ Here a false alert refers to a predicted alert of loss of separation that does not materialize and there is no indication of any controller or pilot intervention action. T-TSAFE and CARTS CA have been compared using a full day of recorded traffic from Southern California TRACON (Terminal Radar Approach Control), including aircraft tracks both associated and unassociated with flightplans. ${ }^{6}$ Flights of the associated tracks include a mixture of visual

\footnotetext{
${ }^{1}$ Aerospace Engineer, AFT 210-6, Moffett Field, CA 94035, huabin.tang-1@ nasa.gov.
} 
and Instrument Landing System (ILS) approach flights. The result shows the number of predicted conflict aircraft pairs common to both T-TSAFE and CARTS CA is small although T-TSAFE was configured to employ alert thresholds based on IFR (Instrument Flight Rules) radar separation standards that are not applicable to visual approaches in practice. This is consistent with an $80 \%$ nuisance-alert rate of CARTS CA concluded by an FAA analysis. ${ }^{7}$ Here a nuisance alert refers to an alert that draws the attention of the controller but fails to provide useful information. ${ }^{7}$ CARTS CA produces a large number of Mode-C Intruder (MCI) alerts, ${ }^{6}$ which involves an associated and an unassociated aircraft. T-TSAFE is found to produce $\sim 50 \%$ less MCI alerts than CARTS CA does. ${ }^{6}$ Furthermore, the result indicates that the expected large number of nuisance alerts from treating visual approach flights as instrument flights could be removed with a high-severity alerting option, ${ }^{6}$ which amounts to an alert threshold of reduced standard separation.

Two concerns need to be further addressed. First, STARS CA is expected to have improved over CARTS CA, so it is interesting to compare T-TSAFE with STARS CA as well. Second, there are seemingly different visual approaches that are performed at TRACON facilities other than Southern California TRACON (SCT). These visual approaches should be examined further.

Visual approaches to various runway configurations are studied in this paper. A set of safety alert thresholds for aircraft conducting visual approaches is proposed based on input of Subject Matter Experts (SMEs) as well as visual approach procedures and common practices. The goal is to maximize the alert thresholds while minimizing nuisance alerts with the usage of flight intent information. Fast-time simulations of T-TSAFE with recorded real-world traffic data from Dallas/Fort Worth (DFW) TRACON (D10) allows performance comparison with STARS CA as well as testing of the safety alert thresholds. The results show T-TSAFE produces far fewer nuisance alerts with a manageable total number of alerts.

The rest of this paper is organized as follows. Section II focuses on the concepts of visual approaches and safety alert thresholds consistent with common practices. Section III describes the experiment and the data used in the simulations. Section IV shows the results of analyzing the experimental output. Section V summarizes the findings.

\section{Visual Approach Procedures, Practice, and Alert Thresholds}

A visual approach is an air traffic control authorization for an aircraft on an IFR flightplan to proceed visually to the airport or runway. ${ }^{3}$ At all times the pilot must have either the airport or the preceding aircraft in sight. Reported weather at the airport must be ceiling at or above $1,000 \mathrm{ft}$ with visibility of 3 statute miles or greater.

A large portion of daily terminal operations involve visual approach aircraft for which controllers are still responsible for providing safety alerts. ${ }^{3}$ Visual approaches help increase capacity under VMC (Visual Meteorological Conditions) weather, but they also complicate separation provision. Before making a visual approach clearance, the controller makes sure the particular aircraft is not at loss of separation with any other aircraft. After the clearance, the pilot becomes responsible for maintaining visual separation with the preceding aircraft on the same or an adjacent parallel runway. Maintaining visual separation with a preceding aircraft approaching the same runway also means maintaining a separation large enough to guarantee safe landing and avoid go-around. For simultaneous approaches to multiple parallel runways, the succeeding visual approach aircraft may not overtake the preceding aircraft if wake turbulence separation violation may occur, as when the runway centerline separation is less than $2500 \mathrm{ft}$. Standard IFR radar separation between the visual approach aircraft and other visual or ILS approach aircraft must still be maintained. In addition, the controller must also provide safety alerts to any aircraft, visual approach aircraft included, that is expected to be placed in unsafe proximity to terrain, obstructions, or other aircraft. ${ }^{3}$ Thus, an automated air traffic system should provide controllers with separation alerts on potential violation of the standard separation, as well as safety alerts to help controllers make decision on potentially unsafe situations when visual approach aircraft are involved.

Safety alerts for visual approach aircraft may help prevent inadvertent errors as well. For example, a visual approach aircraft may follow the wrong preceding aircraft. The lack of standard for alerting and the complexity of various possible visual approaches make it hard to determine proper alert thresholds for safety alerts. The CA functionality of CARTS and STARS issues alerts to controllers when an aircraft gets into dangerous proximity of another aircraft. This may help controllers to provide safety alerts when visual approach aircraft are involved. However, the percentage of nuisance alerts are too large to be useful. ${ }^{7}$ Larger alert thresholds than those of CA may be adopted as well when flight intent information is used, allowing the controllers more time to respond to the situation.

T-TSAFE ${ }^{4}$ predicts separation conflicts between a pair of aircraft using a deterministic kinematic trajectory and the standard separation criteria specified in the FAA Order JO $7110.65 \mathrm{~V} .{ }^{3}$ The aircraft trajectories are based on current states of the aircraft from radar or ADS-B (Automatic Dependent Surveillance - Broadcast) track updates and the available flight intent information. Track history or historic flight path is needed when wake turbulence is involved so 
that one can determine if the trailing aircraft is currently directly behind (within $2500 \mathrm{ft}$ of the flight path) and either at the same altitude or within $1000 \mathrm{ft}$ below the preceding aircraft. Some specific rules for visual approaches have been outlined in FAA Order JO $7110.65 \mathrm{~V},{ }^{3}$ although alert thresholds for visual approach flights are not addressed. Previous work ${ }^{5-6}$ uses a high-severity option, which amounts to alert thresholds reduced from the standard separations, for alerting on visual approach aircraft pairs. For aircraft approaching the same runway, the high-severity option amounts to alerting when the time to the first loss of non-wake (wake) conformance separation of $0.75(0.85)$ is less than 40 seconds, which is a time that is typically sufficient for the controller to respond to potential conflicts in terminal airspace. The conformance separation is defined as $s=\sqrt{V_{r}^{2}+H_{r}^{2}}$ where $H_{\mathrm{r}}=r / r_{\min }$ and $V_{\mathrm{r}}=h / h_{\min }$ are, respectively, the horizontal and vertical separations retained. ${ }^{8}$ Here $r$ and $r_{\min }$ are, respectively, the horizontal separation of the aircraft pair at some instant and the required standard IFR horizontal separation minimum and, similarly, $h$ and $h_{\min }$ are, respectively, the corresponding vertical values. Conformance separations of operational errors of classes A and B correspond to $s \leq 0.75$ for non-wake and $s \leq 0.85$ for wake conflicts. The alert thresholds being related to the severities of operational errors reflect the seriousness of the encounters, and they happen to coincide with the typical separation of visual approach aircraft pairs (to the same runway) in practice. The ultimate test for the alert thresholds should be the time buffer that allows controllers to respond and the nuisance-alert rate from testing against real-world traffic data. For aircraft approaching parallel runways, high severity was defined based on whether an aircraft was to cross the localizer. ${ }^{6}$ This needs generalization to accommodate different visual approaches of aircraft pairs to multiple parallel runways.

\section{A. Approach to the Airport or Runway}

An aircraft can be cleared for visual approach to the airport or runway when the pilot reports the airport or runway in sight and there are no potential losses of separation with any other aircraft at the time. It may also be cleared for visual approach to the runway following the preceding aircraft if the pilot reports sighting the preceding aircraft irrespective of the airport or runway being in sight or not. ${ }^{3}$ Figure 1 shows an example of three aircraft approaching runway 24R at LAX (Los Angeles International Airport). Aircraft A1 is on ILS approach and is referred to as I4R at SCT. Aircraft A2 is cleared for visual approach, referred to as V4R, following and maintaining visual separation from aircraft A1. Aircraft A3 is cleared for V4R as well following and maintaining visual separation from aircraft A2. We will generally refer to this kind of visual approach as VA in what follows.

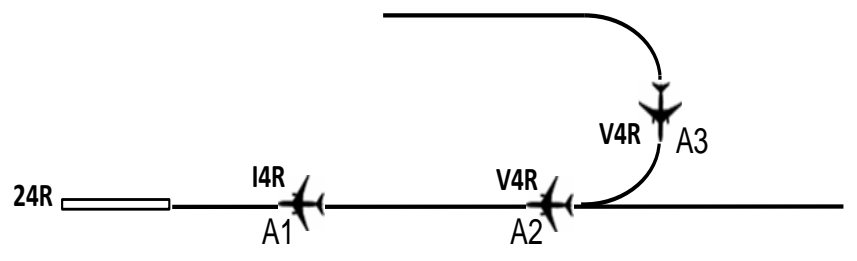

Figure 1. Some examples of visual approach to the airport or runway at LAX.

VA aircraft pairs approach the same runway successively with visual separation, and the separation maintained must be large enough to allow safe landing. Based on input from SMEs, and confirmed from real-world traffic data as well, aircraft pairs on VA typically maintain a separation of about 2.2 NM or more when they are at the same level and when a standard 3 NM ILS separation is required if they are on ILS approaches. This corresponds approximately to the conformance separation of 0.75 for non-wake conflicts. It is at the border of a class B operational error when standard separation applies and may be a good point to draw the attention of the controllers so they may decide if a safety alert to the pilot is necessary. This would be useful to controllers as long as the number of alerts is not excessive and there are not too many false alerts. Testing against real-world traffic data will verify if this is the case. Thus, we propose a safety alert threshold for VA aircraft pairs when a minimum conformance non-wake separation of 0.75 or wake separation of 0.85 is violated within 40 seconds. Again, 40 seconds is a time that is typically sufficient for controllers to respond to a potential conflict in terminal airspace. Fine tuning of the thresholds can be done in the actual system as long as it is within the safe practice and no proliferating nuisance alerts are generated.

\section{B. Approach to Parallel Runways}

Visual approaches to parallel runways are generally described in Section 7-4-4 of FAA Order JO 7110.65V. ${ }^{3}$ Here the relevant procedures for different separations of runway centerlines from Ref. 3 are summarized while the safety thresholds for generating alerts to the controllers are discussed.

\section{Runways Separated by Less Than 2500 Feet}

An aircraft may be cleared for visual approach to a runway following, and maintaining visual separation with, a preceding aircraft that approaches an adjacent runway less than $2500 \mathrm{ft}$ apart. Once the preceding aircraft, which may 
be on visual or ILS approach, is established on the final, the succeeding aircraft may close up. ${ }^{3}$ However, a heavy or B757 aircraft is not permitted to overtake another aircraft, and a large aircraft is not permitted to overtake a small aircraft.

Figure 2 illustrates an example of aircraft approaching parallel runways with centerline separation less than $2500 \mathrm{ft}$. The aircraft approach runway $24 \mathrm{R}$ and $24 \mathrm{~L}$ at LAX. Aircraft A2 follows ILS-approach aircraft A1 on the adjacent runway $24 \mathrm{R}$ on a visual approach to runway $24 \mathrm{~L}$. Aircraft A3 follows $\mathrm{A} 2$ on visual approach to runway $24 \mathrm{~L}$, and aircraft A4 is on V4R following aircraft A3 on the adjacent runway. Aircraft A2 may close up to aircraft A1 such that both aircraft approach their runways almost side by side. However, if wake turbulence applies, the succeeding aircraft would need to be

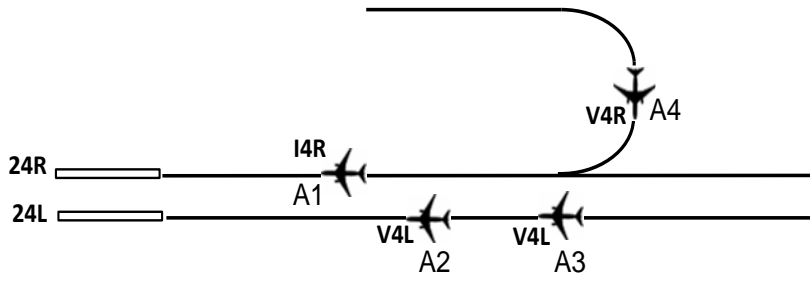

Figure 2. Visual approach to parallel runways less than $2500 \mathrm{ft}$ apart at LAX. above the flight path of the preceding aircraft.

To provide safety alerts to the controllers, we need to determine proper safety alert thresholds, which may be rationalized based on the procedures summarized above. Since runways less than $2500 \mathrm{ft}$ apart are treated as the same runway for ILS approaches, before the visual approach aircraft pair is established on their localizers, we should use the single runway safety thresholds, i.e. non-wake and wake conformance thresholds of 0.75 and 0.85 respectively and less than 40 seconds time to potential conflicts. Once they are established on their finals, alerts are generated only when a blunder is expected to occur. A blunder is defined here as a situation in which an aircraft is off and is crossing the localizer toward the other runway. A safety alert threshold that may predict and detect a blunder is a conformance alert threshold of value $d / r_{\min }$ for aircraft off the localizer, where $d$ is the runway centerline separation and $r_{\min }$ is the required standard IFR horizontal radar separation. Heavy or B757 aircraft overtaking another aircraft or large overtaking small will be predicted because standard separation alerts will be generated as a result of the violation of wake turbulence standards. We should note that we have not observed a real-world encounter for visual parallel approaches, with runway centerlines less than $2500 \mathrm{ft}$ apart, in which the succeeding aircraft pulls up too close to the preceding aircraft. They typically maintain a separation that conforms with the conformance thresholds of 0.75 or 0.85 instead.

Simultaneous ILS approaches to parallel runways separated by less than $2500 \mathrm{ft}$ apart may be conducted, but the runways would be treated as a single runway as far as standard separation is concerned. However, an aircraft may be cleared for ILS approach to a runway while maintaining visual separation with the preceding aircraft on the adjacent runway when the pilot reports the preceding aircraft in sight. In this case, the succeeding aircraft is said to be on VV. Figure 3 shows such a situation. Aircraft A1, A2, and A3 are on ILS approach to runway 24R, and aircraft A4, A5, and A6 are on ILS approach to runway 25L. However, aircraft A5 maintains a visual separation with aircraft A2 since it is on VV. Note that aircraft on VV implies it is on ILS approach to the runway. Thus, standard separation thresholds apply to aircraft A5 with any aircraft other than A2, and only high-severity alerts

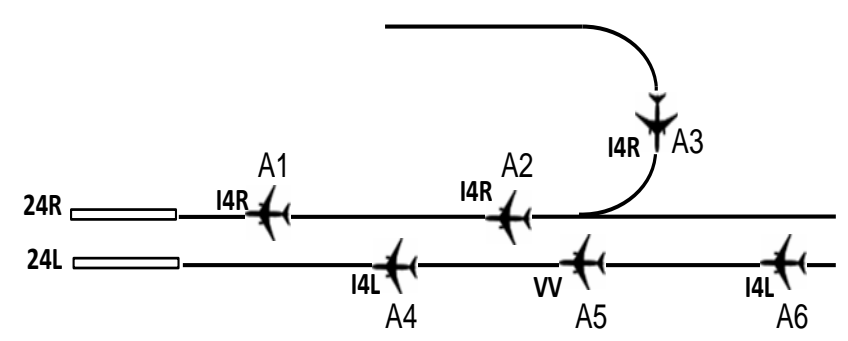

Figure 3. ILS approach mixed with visual VV approach to parallel runways less than $2500 \mathrm{ft}$ apart. may be generated for the A5 and A2 pair.

\section{Parallel Runways Separated by 2500 to 4300 Feet}

Visual approaches may be conducted simultaneously to parallel runways separated by 2500 to 4300 feet. According to FAA Order JO $7110.65 \mathrm{~V},{ }^{3}$ standard separation needs to be provided until the aircraft are established on a course heading that will intercept the extended centerline of the runway at an angle not greater than 30 degrees. Thereafter it is not necessary to apply any other type of separation with aircraft on the adjacent final approach course. Thus, each runway localizer may have a stream of visual approach aircraft, and there will not be any alert unless there is a blunder in which an aircraft is off the localizer and attempts to cross to the other runway.

Thus, the appropriate safety alert thresholds in this case may be as follows. Before turn-on to the localizers, highseverity conformance threshold of 0.75 is used with the time to potential conflicts being less than 40 seconds. Once 
both aircraft intercept their localizers with course headings within 30 degrees of the extended centerlines of the runways, the threshold reduces to $d / r_{\min }$ and is applied only if one aircraft drifts off the localizer.

\section{Parallel Runways Separated by More Than 4300 feet}

Visual approaches may be conducted simultaneously to parallel runways separated by 4300 feet or more. The requirement is merely that each aircraft must be assigned a heading that will allow the aircraft to intercept the extended centerline of the runway at an angle not greater than 30 degrees. ${ }^{3}$ It is not necessary to apply any other type of separation with aircraft on the adjacent final approach course. Thus, the parallel runways are operated almost independently like two separate airports, which are called "complexes" at SCT. In what follows, we will use this terminology generally.

Figure 4 shows multiple aircraft approach simultaneously runways $24 \mathrm{R}$ and $25 \mathrm{~L}$ at LAX, and they are thus in different complexes. Aircraft A1 is on ILS approach to runway 24R. Aircraft A2 follows aircraft $\mathrm{A} 1$, and aircraft $\mathrm{A} 3$ follows aircraft A2, on visual approach to runway $24 R$. Aircraft A4 is on visual approach to the airport while aircraft A5 is on ILS approach to runway $25 \mathrm{~L}$. The aircraft streams in different complexes usually join the localizers from different sides of the runways at LAX. SMEs suggest that the controllers do not want to see any alerts between a VA aircraft in one complex and an ILS approach or VA aircraft in another complex, even during a turn-on to the localizer, unless a blunder

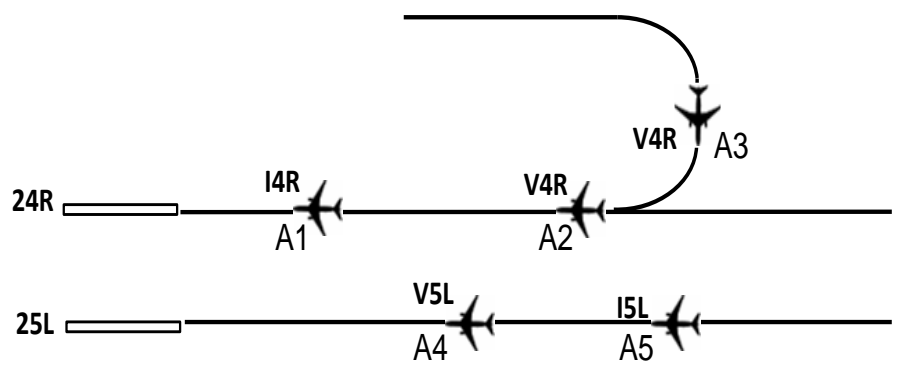
is expected to occur. ${ }^{5}$

It does not occur frequently that a pair of visual approach aircraft approaches runways in different complexes at LAX from the same side. However, in general, aircraft on visual approaches often approach from same or different sides of the runways simultaneously. Once the aircraft are established on their finals, there should not be any safety alerts generated unless a blunder is expected to occur, because the aircraft operate independently in different complexes. But before they are established, when they turn onto their localizers from the same side of the runways, or when their flight paths cross each other when they turn onto the localizers from different sides of the runway, some safety threshold should be used to alert the controllers of potentially unsafe encounters. Actual examples of these situations are discussed in Sec. IV. The safety threshold we propose to use is the conformance alert threshold of value equal to $\min \left\{d / r_{\min }, 0.75\right\}$, where $d$ is the runway separation and $r_{\min }=3 \mathrm{NM}$ is the standard horizontal separation. For example, if the runway separation is $9000 \mathrm{ft}$, the conformance alert threshold would be 0.5 . This threshold applies when the aircraft blunders after being established on the final or during the aircraft's turn-ons to their localizers. The value of 0.75 here accounts for possible large runway separations. Note that, if the aircraft turn on to their localizers from different sides of the runways and their flight paths do not cross, the safety threshold will never be triggered unless one aircraft is predicted to overshoot the localizer. We should also note that alerts could still be generated for each independent complex based on the safety thresholds discussed earlier.

An aircraft may be cleared for ILS approach to a landing runway and maintain visual separation with a preceding visual or ILS approach aircraft in an adjacent runway more than 4300 feet apart. This situation is called VS. Figure 5 shows the possible simultaneous four-runway visual or ILS approaches on the 24 and 25 runways at LAX. Aircraft A1 and A2 are on ILS approaches to runway $24 R$ and $25 R$, respectively. Aircraft A3 is on V4R following aircraft A1, while aircraft A4 is on ILS approach to runway $25 \mathrm{~L}$ maintaining visual separation (VV) with aircraft A2. Aircraft A5 is on ILS approach to runway 24R, while aircraft A6 is on ILS approach to runway $25 \mathrm{~L}$ and maintaining visual separation (VS) with aircraft A5. In general,

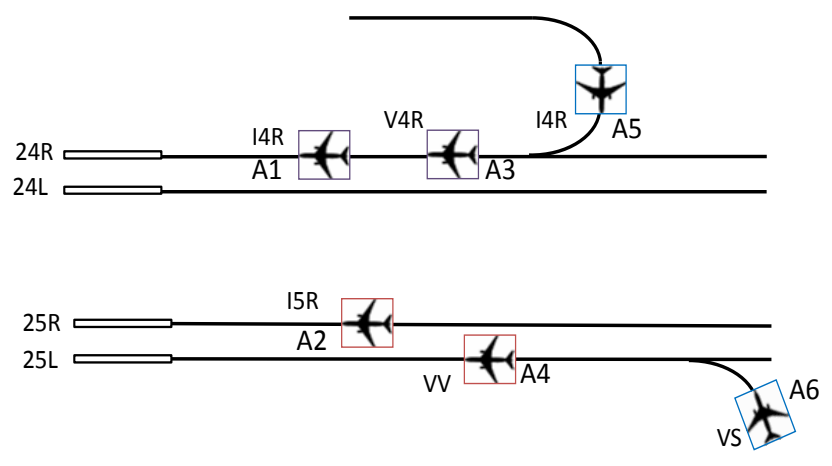

Figure 5. Some visual approach pairs in two complexes at LAX including a visual VS approach pair. 
the VS aircraft pairs could be approaching the final approach courses from the same side of the runways, although at LAX they usually approach the runways from the opposite sides. The same safety threshold discussed above applies for the VS aircraft pair. For example, when they turn on to their localizers from different sides of the runways with no crossing of flight paths as in Fig. 5, no alerts will be generated during turn-on to the localizer unless an overshoot is predicted to occur.

\section{Safety Alert Threshold Comparison}

Multiple-runway visual approaches with a mix of visual and ILS approaches can be highly complex as in the case of the four-runway two-complex simultaneous approaches at LAX. There is also a variety of safety alert thresholds discussed so far. Thus, we summarize and compare them with those of CA in Table 1 below. STARS and CARTS CA uses smaller alert thresholds corresponding to $d=0.7$ times the runway centerline separation for parallel runway approaches; otherwise CA typically uses $\sim 1 \mathrm{NM}$ horizontal separation and $\sim 300 \mathrm{ft}$ vertical separation, which amounts to a conformance threshold of approximately 0.45 . In Table 1 , we have denoted the time to first conflict or violation of the conformance threshold as $t_{\mathrm{c}}$. For parallel runways with separation between $2500 \mathrm{ft}$ and $4300 \mathrm{ft}$, the $30^{\circ}$ turn-on means a turn-on with course headings within $30^{\circ}$ of the extended centerlines of the runways. Note again that blunder means an aircraft is off the localizer and is heading toward the other parallel runway. Thus, two aircraft turning onto their localizers from the same side would not constitute a blunder unless they overshoot. As seen in Table 1, our proposed safety alert thresholds are relatively larger than those of CA. As a result, controllers may have longer time to respond to such situations. With flight intent information taken into consideration, T-TSAFE also yields larger alert lead times. Thus, T-TSAFE may have larger safety buffers. More tests against real-world traffic data may help refine the safety alert thresholds. In the next section, we use the air traffic data from D10 on a specific day that involves mostly visual approach flights to show that our larger safety thresholds counter-intuitively yield fewer nuisance alerts and a smaller total number of alerts.

Table 1 Safety Alert Conformance Thresholds for T-TSAFE and CA

\begin{tabular}{|c|c|c|c|c|c|c|c|}
\hline \multirow{3}{*}{ Setup } & \multicolumn{2}{|c|}{ Single Runway } & \multicolumn{5}{|c|}{ Parallel Runways of Separation $d$} \\
\hline & \multirow[b]{2}{*}{ wake } & \multirow[b]{2}{*}{$\begin{array}{l}\text { non- } \\
\text { wake }\end{array}$} & \multicolumn{2}{|c|}{$d<2500 \mathrm{ft}$} & \multicolumn{2}{|c|}{$2500 \mathrm{ft}<d<4300 \mathrm{ft}$} & \multirow{2}{*}{$\begin{array}{c}d>4300 \mathrm{ft} \\
\text { not both on localizers }\end{array}$} \\
\hline & & & $\begin{array}{l}\text { prior to and } \\
\text { during turn-on }\end{array}$ & blunder & $\begin{array}{l}\text { prior to } 30^{\circ} \\
\text { turn-on }\end{array}$ & blunder & \\
\hline \multirow{2}{*}{ T-TSAFE } & 0.85 & 0.75 & 0.75 & $d / r_{\min }$ & 0.75 & $d / r_{\min }$ & $\min \left\{d / r_{\min }, 0.75\right\}$ \\
\hline & \multicolumn{2}{|c|}{$t_{\mathrm{c}}<40$ seconds } & \multicolumn{5}{|c|}{$t_{\mathrm{c}}<40$ seconds and no alerts when both aircraft are on the localizers } \\
\hline CA & \multicolumn{2}{|c|}{0.45} & \multicolumn{5}{|c|}{$0.70 \mathrm{~d} / r_{\min }$} \\
\hline
\end{tabular}

\section{Experiment}

Fast-time simulation T-TSAFE experiments with real-world air traffic data from D10, including STARS data, were performed. Though most of the DFW arriving flights in the data were on visual approaches, the simulations were done by assuming the associated flights, with their tracks associated with flightplans, to be conducting first ILS and then visual approaches. The performance of T-TSAFE was evaluated by studying the generated MCI and non-MCI alerts in comparison with those of STARS CA.

To study the full benefits of T-TSAFE, we use air traffic data with both associated and unassociated aircraft as well as the flightplan information for the associated aircraft. Recorded real-world traffic data of July 26, 2014 from D10 were used. Clear VMC weather at Dallas Fort Worth on that day and the fact that all final approach courses were short indicate that most, if not all, arrival aircraft were making visual approaches to the airports or runways. Air traffic data recorded at NASA currently includes radar tracks and flightplan information of associated aircraft but not unassociated tracks. FAA's STARS CDR (Continuous Data Recording) data contains CA conflicts and associated as well as unassociated tracks but no flightplan information. Thus, we extracted unassociated tracks from the STARS CDR data and inserted them into the NASA-recorded data. Unassociated tracks with negative and zero altitudes were excluded. Inferred altitude clearances ${ }^{4}$ or temporary level-off altitudes for the associated flights were added to simulate altitude amendments in the recorded traffic data as if the level-off intent was available. This yields a complete set of associated and unassociated tracks with flightplan and altitude clearance intent information for the associated flights. 
In practice, only altitude clearances for conflict resolutions are needed, as altitude restrictions for waypoints on typical terminal procedures are available.

Fast-time T-TSAFE simulations of the traffic data were performed with a real-time preprocessor of radar tracks as in Ref. 6. When an aircraft enters the TRACON, its track may get associated with a flightplan and become an associated track. On occasion, both the associated and unassociated tracks for the same aircraft may coexist for some period of time. As a result, CA confuses the associated and unassociated tracks as being two separate aircraft and therefore generate a conflict alert. This kind of "self alert" is clearly a false MCI alert that should not be issued to the controller. CA also generates alerts in which the identical associated and unassociated tracks cause conflicts with another aircraft simultaneously. The MCI alerts from the unassociated tracks are then redundant alerts. The preprocessor processes the unassociated tracks in real time without knowledge of future tracks. It may associate unassociated tracks with associated tracks by stitching based on position and velocity to avoid the self and redundant alerts. A table that maps unassociated and associated tracks and sometimes two unassociated tracks is generated by the preprocessor, which helps to identify STAR CA's MCI conflict pairs extracted from STARS CDR data.

Post processing of the XML outputs of T-TSAFE allows generation of statistics of T-TSAFE MCI and non-MCI alerts. Past analyses using recorded real-world air traffic data have assumed all arriving flights to be conducting ILS approaches because visual approach intent information is generally not available. ${ }^{4}$ Analysis has also been done from fast-time simulation of recorded air traffic data from Human-In-The-Loop experiments. ${ }^{5}$ The results of both analyses determine the false-alert rates are $\sim 10 \%$ for T-TSAFE when ILS approaches are assumed. Knowing the false-alert rate of T-TSAFE will allow estimation of the nuisance-alert rate of STARS CA. Furthermore, assuming the visual approaches as ILS approaches will increase the number of separation alerts and thus increase the alert pairs common to both T-TSAFE and STARS CA. Examination of these common alert pairs will test the safety alert thresholds, showing why they may be valid separation alerts but may not be good safety alerts when visual approaches are taken into account. Thus, we performed simulations assuming the arriving flights as either ILS or visual approaches. However, we would not be able to identify when the visual approaches were issued. This amounts to the assumption that there were no violations of the standard separations, or the number of violations is negligibly small, before the visual approaches were issued.

\section{Results}

The alerts on conflict pairs for T-TSAFE are analyzed and compared with those of STARS CA for two scenarios: (1) when all the DFW arrivals are assumed to be on ILS and (2) when they are assumed to be on visual approaches. In making the comparison, the alerts for T-TSAFE and STARS CA are classified into Loss of Separation (LOS), nonLOS, and non-T-TSAFE alert pairs for both MCI and non-MCI alerts. If the predicted loss of separation actually materializes later, the alert is called an "LOS alert". On the other hand, if the LOS prediction is not followed by an actual loss of separation, the alert is called a "non-LOS alert". A non-LOS alert may be a valid rather than false alert, as there could have been controller or pilot intervention after the alert was generated. For safety alerts, we still use this classification with the understanding that an LOS alert would mean the aircraft pair was predicted to violate the safety thresholds and was later detected to actually violate those thresholds. From a different perspective, the alerts are also grouped into DFW and non-DFW alerts. If at least one aircraft arrives into or departs from DFW, the alert pair is called a DFW alert pair, otherwise it is a non-DFW alert pair. For convenience, the alert pairs that are common to both T-TSAFE and STARS CA are referred to as "common conflict pairs".

\section{A. ILS Approach Simulation}

In this simulation and analysis, no visual approach intent information is assumed and all arriving flights to DFW are assumed to be on ILS approaches, so the standard separation thresholds apply.

\section{Alert Rate}

The alert rate in terms of the number of conflict pairs per hour was determined and analyzed. It is expected that most DFW arriving flights violate the standard separation because the input data contains aircraft that were mostly conducting visual approaches. By assuming all aircraft conducting ILS approaches, T-TSAFE generates valid separation alerts with about $10 \%$ false alert rate. ${ }^{4,5} \mathrm{By}$ increasing the number of separation alerts and studying the conflict pairs that are common to both T-TSAFE and CA, we can estimate the nuisance-alert rate of CA.

Figure 6 shows the alert rates per hour for T-TSAFE and STARS CA classified in terms of LOS, non-LOS, and non-TTSAFE alert pairs for both MCI and non-MCI alerts. Also indicated in the different STARS CA alert bars are the proportions of common conflict pairs. As in the case of CARTS CA, ${ }^{6}$ the common conflict pairs are only a small percentage of the total STARS CA alerts. If we assume a small percentage of T-TSAFE alerts are false, the percentage 
of false alerts for STARS CA will still be large. For non-MCI alerts, the common conflict pairs are $21 \%$ of the total non-MCI alerts. The other $79 \%$ of STARS non-MCI alerts are completely different pairs from those of T-TSAFE non-MCI alerts. Since T-TSAFE is able to detect any LOS by design, ${ }^{4}$ the $79 \%$ non-common conflict pairs for STARS CA would not be at losses of separation when the alerts were predicted. The time to the predicted conflicts would thus be about 40 seconds if we consider STARS CA predicts conflicts with $\sim 1 \mathrm{NM}$ alert threshold and an approach speed of $\sim 200$ knots. This time is on the border of the look-ahead time of CA and thus the alerts have large possibility of being nuisance. Considering a $10 \%$ false alert rate for T-TSAFE, ${ }^{4,5}$ it is conceivable that the non-common conflict pairs of STARS CA would have a nuisance-alert rate of $90 \%$. Then, the total nuisance-alert rate of STARS CA non-MCI alerts could be estimated as $(0.1 * 21 \%+0.9 * 79 \%)=73 \%$, which is about $10 \%$ improvement over CARTS CA. Similarly deduced $80 \%$ nuisancealert rate for CARTS $\mathrm{CA}^{5}$ was consistent with the FAA analysis. ${ }^{7}$ The number of MCI alerts for STARS CA is also significantly smaller than that for CARTS CA, although one should be careful in making comparison here, since SCT has more airports, especially small airports where MCI alerts dominate. The MCI alerts for T-TSAFE are only about $24 \%$ of those STARS CA alerts, a $76 \%$ reduction. This is a bigger reduction than the $55 \%$ for SCT. ${ }^{6}$ The common conflict pairs for MCI alerts of STARS CA constitute about $10 \%$ of the total MCI alerts of STARS CA.

Figure 6 also shows that the number of T-TSAFE non-MCI alerts is significantly more than that of STARS CA alerts, whereas similar comparison with CARTS CA was not as significant. ${ }^{5}$ This difference is attributed to the fact that essentially all of the DFW arrivals were making visual approaches, while the ratio of visual to ILS approaches in the SCT data was not as high. The number of non-MCI alerts depends on the degree of mixing of visual and ILS approaches in the input traffic data.

Figure 7 shows the alerts per hour for both T-TSAFE and STARS CA, grouped by MCI and non-MCI as well as DFW and non-DFW alerts. The T-TSAFE non-MCI bar indicates that about $97 \%$ of T-TSAFE non-MCI alerts are DFW alerts, whereas the T-TSAFE MCI bar indicates that only about $12 \%$ of T-TSAFE MCI alerts are DFW alerts. On the other hand, the CA non-MCI bar indicates that about $40 \%$ of CA nonMCI alerts are DFW alerts whereas the CA MCI bar indicates that about $3 \%$ of CA MCI alerts are DFW alerts. The large percentage of DFW non-MCI alerts for T-TSAFE is expected, as there are a lot more DFW arrivals than other small airports in D10 and those arrivals are on visual approaches and thus would violate the standard separation thresholds. The percentage of DFW alerts for STARS CA is relatively small compared with the non-DFW alerts, indicating there might be some suppression of DFW alerts. The small percentage CA MCI DFW alerts also suggest that small airports are more susceptible to MCI alerts.

To summarize, in this ILS approach simulation using mostly visual approach traffic data as input, due to the use of flight intent information, T-TSAFE is able to predict the large number of expected conflicts with a false alert rate of $\sim 10 \%$ based on previous analyses. ${ }^{4,5}$ On the other hand, as would be expected, STARS CA does not help the controllers in aircraft separation. The alert pairs generated by STARS CA are mostly different from those of T-TSAFE, and the nuisance-alert rate is estimated to be about 70\%. The flight intent information T-TSAFE used also reduced the MCI alerts by $76 \%$ as compared to STARS CA.

American Institute of Aeronautics and Astronautics 


\section{Common Conflict Pairs}

As discussed above, only about $21 \%$ of non-MCI pairs alerted by STARS CA were also alerted by T-TSAFE. More specifically, among 83 STARS CA non-MCI pairs, there were 17 pairs that also got alerted by T-TSAFE. These 83 pairs included 18 pairs that STARS CA identified as MCI alerts but that were actually non-MCI alerts. They contained 14 pairs of redundant alerts and 4 pairs of self alerts.

Further examination of the tracks of the 17 common conflict pairs would help understanding the nature of the conflicts and how the flightplan and visual approach intent information as well as the safety alert thresholds in TTSAFE may eliminate many of these alerts and the majority of other non-MCI alerts. The common conflict pairs may be grouped into parallel-runway and non-parallel-runway pairs. The conflict pairs involving arriving flights approaching parallel runways are parallel-runway pairs. Parallel-runway pairs can be further classified into three kinds, depending on whether they approach the runways from opposite sides without track crossing, from the same side, or from opposite sides with track crossing. Among the 17 common conflict pairs, 14 pairs are parallel-runway and three are non-parallel runway pairs.

There were seven common conflict pairs that were parallel-runway pairs approaching from opposite sides of the runways without track crossing. Figure 8 shows an example of such conflicts. Aircraft $\mathrm{ABC} 128$ conducted a visual approach to runway $18 \mathrm{R}$ at DFW while aircraft $\mathrm{ABC} 1390$ simultaneously conducted a visual approach to runway $17 \mathrm{C}$. The separation between runways $18 \mathrm{R}$ and $17 \mathrm{C}$ is $1.5 \mathrm{NM}$ or $9100 \mathrm{ft}$. The squares indicate the waypoints for the nominal interior routes (NIRs). ${ }^{4}$ NIRs are prescribed nominal TRACON paths from the arrival meter fixes down to the runways. They have been used in past air traffic automation efforts ${ }^{9}$ and are in the adaptation of the Traffic Management Advisor (TMA), ${ }^{10}$ a time-based automated tool used to maximize airport efficiency. The circles are $3 \mathrm{NM}$ in diameter and represent the point of first LOS. The stars represent time progression in one-minute intervals near the loss of separation. Aircraft ABC128 was descending below $4000 \mathrm{ft}$ while aircraft ABC1390 leveled off at $3000 \mathrm{ft}$ temporarily (not shown), so they had lost the $1000 \mathrm{ft}$ vertical separation standard and were not yet established on their localizers. Thus T-TSAFE predicts a loss of separation based on the standard separation thresholds about 84 seconds before the first LOS. The inset provides a closeup view of the region near the point of loss of separation, showing that $\mathrm{ABC} 128$ crosses over the localizer (solid-line track) slightly. The safety alert thresholds discussed earlier may allow elimination of many of these parallel-runway alerts when the localizer overshoot is small and the other aircraft has sufficient separation. STARS CA alerts were triggered because dead reckoning was used.

There were six common conflict pairs that were parallel-runway pairs approaching from the same side of the runway. Figure 9 shows an example of such conflicts. Aircraft EFG3245 conducted a visual approach to runway $17 \mathrm{~L}$ at DFW while aircraft NOP365 simultaneously conducted a visual approach to runway $17 \mathrm{C}$ from the same side. The

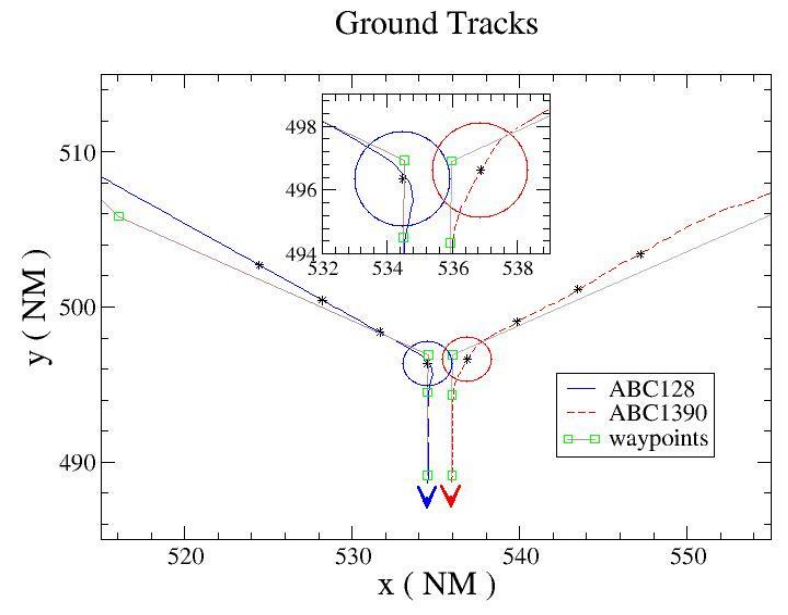

Figure 8. Ground tracks for two aircraft making visual approaches to the finals from opposite sides of the runways.

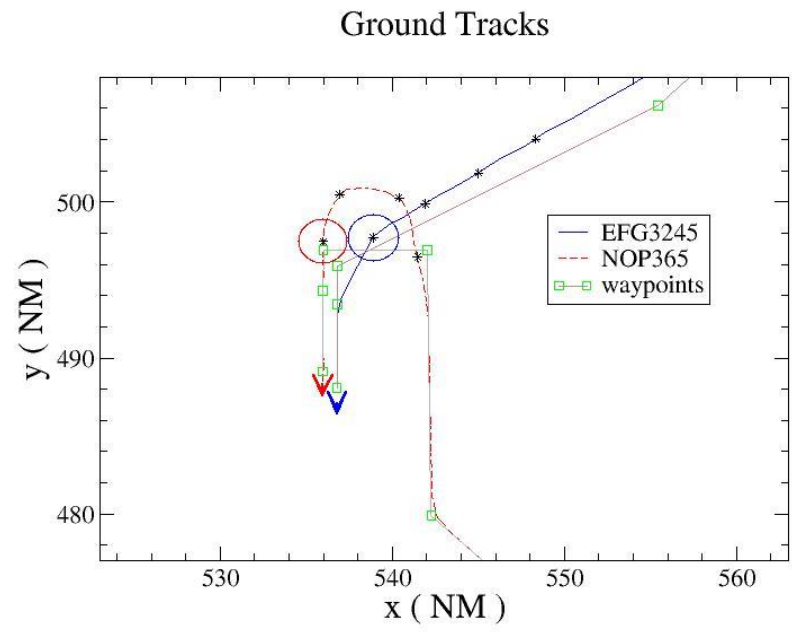

Figure 9. Ground tracks for two aircraft making visual approaches to the finals from the same side of the runways.

9

American Institute of Aeronautics and Astronautics 
separation between runways $17 \mathrm{~L}$ and $17 \mathrm{C}$ is $0.82 \mathrm{NM}$ or $5000 \mathrm{ft}$. Again the squares indicate the waypoints for the NIRs and the circles are 3 NM in diameter and represent the first LOS. The stars represent time progression in one minute intervals near the first LOS. Aircraft EFG3245 was just descending below $4000 \mathrm{ft}$ while NOP365 maintains $3000 \mathrm{ft}$ temporarily (not shown), so they had lost the $1000 \mathrm{ft}$ vertical separation standard while they were not yet established on their localizers. Thus T-TSAFE predicted a loss of separation based on the standard separation thresholds about 75 seconds before the first LOS. However this would be a nuisance alert if one considered that the aircraft were conducting visual approaches; the nuisance alert can be eliminated if we apply the safety alert thresholds. STARS CA generated an alert here even though the alert threshold was only 0.7 of the runway separation because STARS CA relys upon dead-reckoning trajectory predictions.

Figure 10 shows one common conflict pair observed belonging to parallel-runway pairs approaching from opposite sides with track crossing. Aircraft ABC1433 conducted a visual approach to runway $17 \mathrm{R}$ at DFW while aircraft EFG3221 simultaneously conducted a visual approach to runway $18 \mathrm{R}$. The separation between runways $17 \mathrm{R}$ and $18 \mathrm{R}$ is $1.25 \mathrm{NM}$ or $6100 \mathrm{ft}$. Aircraft EFG3221 was maintaining $4000 \mathrm{ft}$ and $\mathrm{ABC} 1433$ was maintaining $3000 \mathrm{ft}$ temporarily while they were turning onto the localizer, but both started to descend before being established on their localizers (not shown). Accordingly T-TSAFE predicted a conflict when they started descending based on the standard separation about 13 seconds before the first LOS. If the visual approach intent information is available, the visual alert thresholds may eliminate this alert as well, even though CA triggers an alert with a horizontal threshold equal to $70 \%$ of the runway separation.

The rest of the three common conflict pairs are non-parallel-runway pairs. Two of them appear to be valid alerts, and one seems to be a false alert. Figure 11 shows the ground tracks for one of the encounters. Aircraft CDE4217 was a flight departing Dallas Love Field (DAL), and aircraft NOP715 was a VFR departure flight from Addison (ADS) airport. RNAV departure routes were not available for either flight. The squares are waypoints from the flightplans. The circles are 1.5 NM in diameter for VFR separation threshold and represent first LOS. Aircraft NOP715 climbed and then leveled at $3300 \mathrm{ft}$. Aircraft CDE4217 climbed to $3000 \mathrm{ft}$ and leveled there (not shown). They lost horizontal separation of 1.5 NM and vertical separation of $500 \mathrm{ft}$. T-TSAFE predicts the LOS with a lead time of 52 seconds. VFR flight NOP715 should have been leveled at $3500 \mathrm{ft}$ to avoid the conflict. Thus, this is a valid alert which both TTSAFE and STARS CA issued.

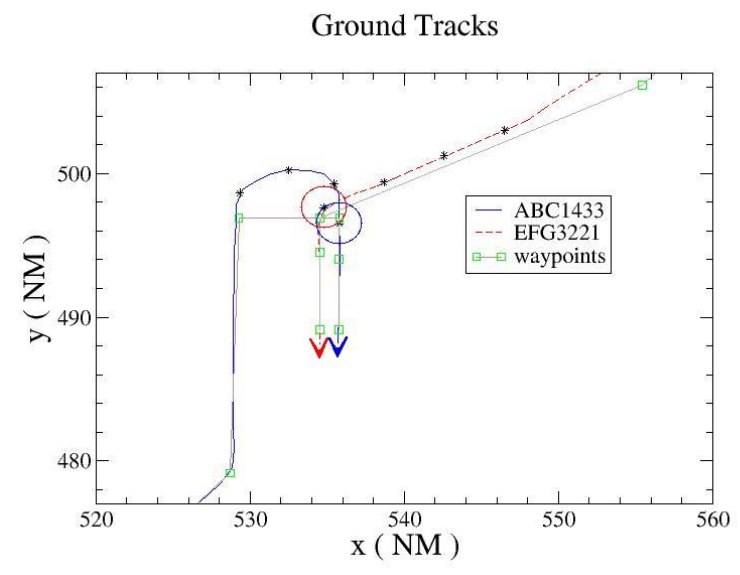

Figure 10. Ground tracks for two aircraft making visual approaches to the finals from opposite sides of the runways with tracks crossed.

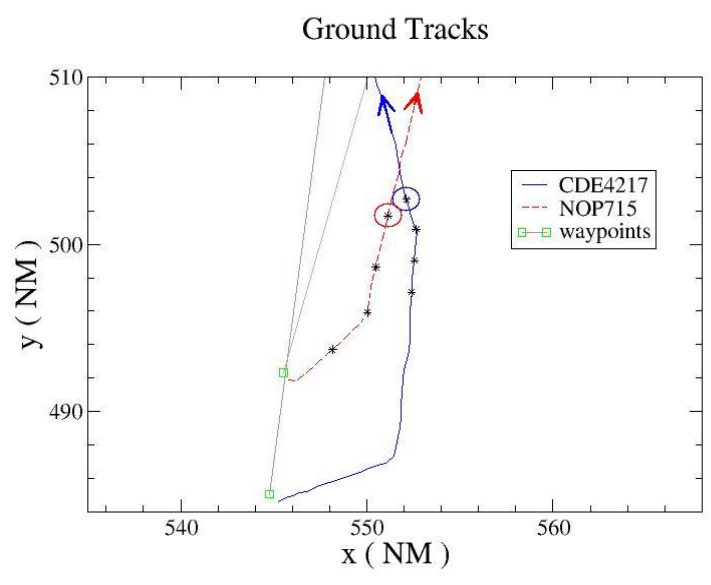

\section{Figure 11. Ground tracks for a conflict pair involving a departure (CDE4217) and another VFR departure (NOP715).}

Figure 12 shows the ground tracks for another non-parallel-runway conflict pair between a departure and an arrival flight. Aircraft NOP330 was an IFR departure from ADS and was climbing from about $1000 \mathrm{ft}$ before the conflict. Aircraft STU4062 was an arrival to DAL and was temperarily level at $3000 \mathrm{ft}$. The circles are $3 \mathrm{NM}$ in diameter. The horizontal and vertical separations were lost briefly before the aircraft entered into diverging courses that resolved the conflict. Aircraft NOP330 did not have an RNAV departure route available, and aircraft STU4062 did not follow its NIR closely in an apparent visual approach to runway 13R at DAL. T-TSAFE alerts about 40 seconds before the first LOS. This is a valid alert which STARS CA issued as well. 
Figure 13 shows the ground tracks for another non-parallel-runway conflict pair between two arriving flights, which turns out to be a false alert. Aircraft EFG3071 was an arrival to DFW on visual approach to runway 17L. Aircraft NOP451 was an arrival to DAL on visual approach to runway $13 \mathrm{R}$. The circles are $3 \mathrm{NM}$ in diameter. The solid lines are the predicted trajectories. The aircraft were both descending at the same altitude. As can be seen, the actual tracks never lose horizontal separation of 3 NM, so this was a false alert. This false alert was due to an inaccurate prediction of the horizontal trajectory of aircraft NOP451 as a result of assuming visual as ILS approach. For ILS approach, it has been assumed that the aircraft attempts to avoid intercepting the localizer too close to the final approach fix.

Examination of the encounters in the above figures shows that, for most cases, T-TSAFE provides proper separation alerts with good lead times. On the other hand, the alerts provided by STARS CA based on dead reckoning and smaller thresholds would be too late to be useful for separation. Furthermore, even many of these common conflict pairs are likely nuisance alerts if we take into account the fact that the flights are conducting visual approaches. As shown next, with flight intent information including visual approaches, proper safety alert thresholds could be designed to eliminate many of these nuisance alerts in addition to other T-TSAFE alerts, which would be nuisance when visual approaches are considered.

\section{B. Visual Approach Simulation}

In this simulation and analysis, all arriving flights to DFW are assumed to be conducting visual approaches with the safety alert thresholds applied. This is consistent with the clear VMC weather and the short final approach courses observed in the recorded traffic data. It is assumed that no loss of separation occurs before the visual approach clearances are issued.

\section{Alert Rate}

Figure 14 shows the alerts per hour for TTSAFE separated into LOS and non-LOS alert pairs. As mentioned earlier, an LOS alert here should mean the aircraft pair was predicted to violate the safety thresholds and was later detected to actually violate those thresholds. Compared with Fig. 6, the number of non-MCI alerts is less by 92\%. The STARS CA results are also shown with the common conflict pairs indicated as well. As expected, there is no change in the percentage of common conflict pairs for the MCI alerts. The

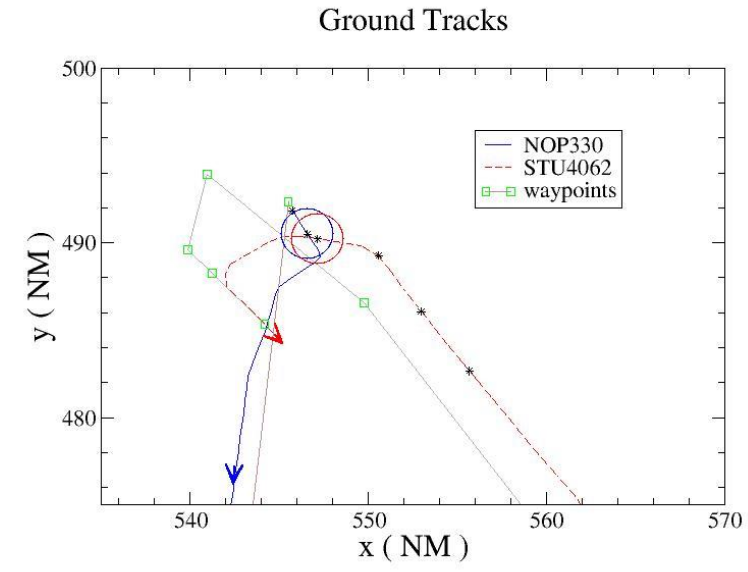

Figure 12. Ground tracks for a conflict pair involving an IFR departure (NOP330) and an arrival (STU4062).

\section{Ground Tracks}

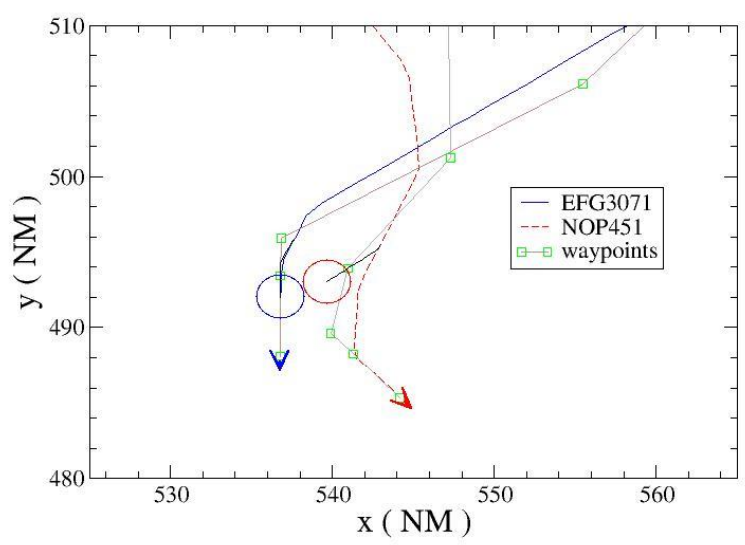

Figure 13. Ground tracks for a conflict pair involving a DFW arrival (EFG3071) and a DAL arrival (NOP451).

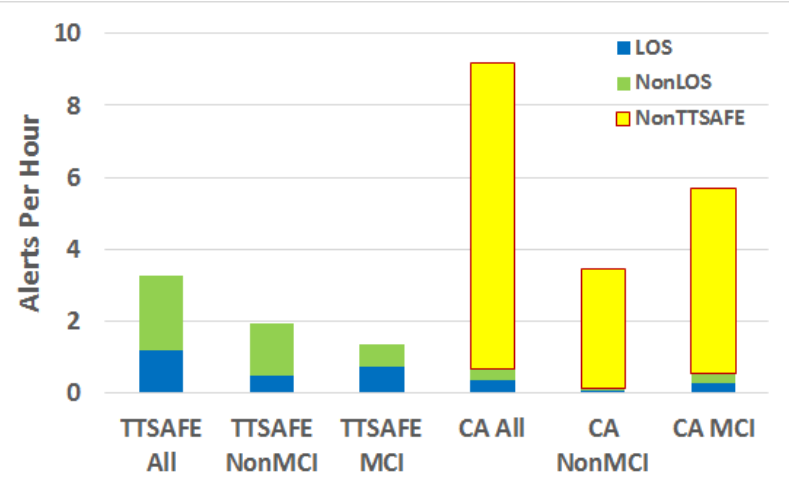

Figure 14. TTSAFE and STARS CA alerts in terms of LOS, non-LOS, and non-TTSAFE when visual approach intent information and safety alert thresholds are used. 
percentage for the non-MCI alerts are considerably lower, as most of the T-TSAFE non-MCI alerts involving DFW arrivals have been eliminated. The number of alert pairs for T-TSAFE are considerably fewer than those of STARS CA for both MCI and non-MCI alerts. This result should dispel the notion that T-TSAFE might not improve much over CARTS CA as some have concluded from Refs. 5 and 6 due to the seemingly high T-TSAFE non-MCI bar in figures similar to Fig. 6 . The result also shows the effectiveness of the flight intent and the safety alert thresholds.

\section{Common Conflict Pairs}

It is interesting to see what effects the safety alert thresholds have on the previous 17 common conflict pairs in the ILS approach simulation experiment examined in Sec. IV.A.2. It turns out only one parallel-runway alert pair and the three non-parallel-runway alert pairs survive. This is the case even though the safety alert thresholds for T-TSAFE is larger than those of STARS CA. The non-parallel-runway pairs are expected to survive since they do not involve visual approach DFW flights. This result shows that 14 of the parallel-runway pairs were reduced to just one, a $93 \%$ reduction.

Figure 15 shows the ground tracks for the common parallel-runway conflict pair that remains in the visual approach simulation experiment. The solid and dashed curves are the actual ground tracks of aircraft ABC128 and ABC1390, respectively, with the arrows indicating the directions of flight. The squares are the waypoints, with the bottom two waypoints being the runway thresholds for runways $18 \mathrm{R}$ and $17 \mathrm{C}$. The dark solid lines are the predicted trajectories for the two aircraft starting from their current positions to the predicted positions of violation of the safety alert thresholds. The circles are both of diameter 1.5 NM, the separation between the two parallel runways. At the moment, aircraft $\mathrm{ABC} 1390$ was still levelling at $3000 \mathrm{ft}$ temporarily while aircraft $\mathrm{ABC} 128$ was descending at about $300 \mathrm{ft}$ higher. There were only two predictions and no actual violations of the safety thresholds were observed as aircraft $\mathrm{ABC} 128$ quickly turned back to the localizer and started descending. Ultimately it is up to the controllers to decide whether this is a valid or nuisance alert, because even though aircraft $\mathrm{ABC} 128$ did overshoot the localizer, it is unlikely to cause any action by the controller. However, should ABC 128 continue to blunder, this alert would be valuable. While the safety alert thresholds should be tuned further in real applications based on the total number of alerts tolerable to the controllers, they are certainly a good starting point.

\section{Other parallel-runway alerts}

T-TSAFE also generated two additional parallelrunway DFW alert pairs that STARS-CA did not alert. It is interesting to examine the validity of these parallel-runway alerts as well.

Figure 16 shows the ground tracks for a pair of DFW arrivals, $\mathrm{ABC} 1458$ and $\mathrm{ABC} 2516$, making visual approaches to runways $17 \mathrm{C}$ and $18 \mathrm{R}$ respectively from the same side. The solid and dashed lines are the actual trajectories of aircraft $\mathrm{ABC} 1458$ and $\mathrm{ABC} 2516$, respectively. The stars are positions separated one minute apart leading to the positions of horizontal closest approach. The squares are waypoints of the NIRs leading to the runway thresholds near the arrows, which indicate the direction of flight. The dark solid lines are predicted trajectories of the aircraft from their current poisitions

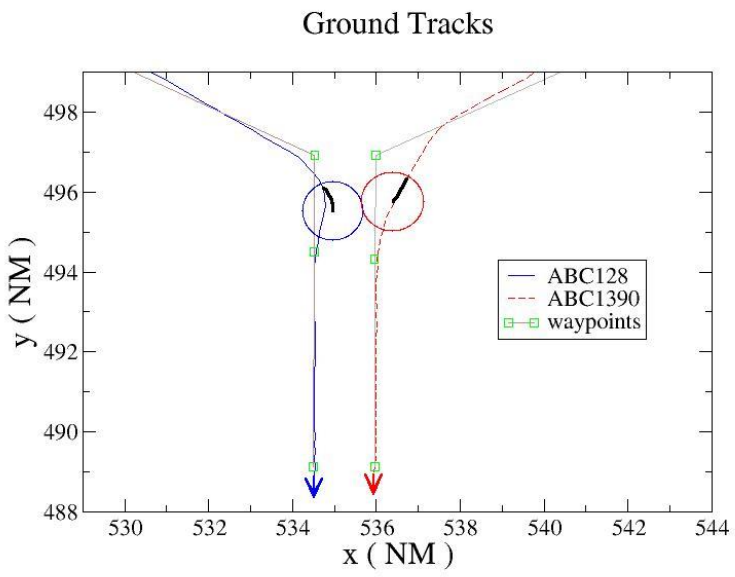

Figure 15. Real and predicted ground tracks for an arrival pair making visual final approaches to runways $18 R$ and $17 C$.

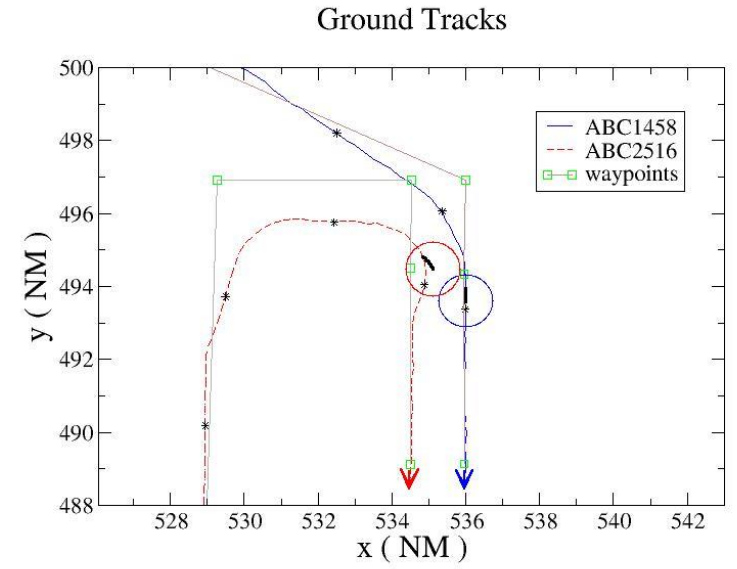

Figure 16. Ground tracks for an arrival pair making visual final approaches from the same side of the runways.

12

American Institute of Aeronautics and Astronautics 
to the first predicted violation of the visual conformance alert threshold of 0.5 . The predicted violation positions are the center of the circles of diameter $1.5 \mathrm{NM}$, corresponding to the runway separation. The alert lasted two radar update cycles and disappeared quickly as aircraft $\mathrm{ABC} 2516$ turned back to the localizer. The aircraft never actually violated the conformance alert threshold of 0.5. This is in the same gray area as Fig. 15. Aircraft ABC2516 drifted off the localizer significantly, but the alert did not last long as the aircraft quickly turned back to the localizer. STARS CA did not alert for this pair due to its smaller alert thresholds.

Figure 17 shows the ground tracks of aircraft $\mathrm{ABC} 1213$ and $\mathrm{ABC} 2371$, which conducted final approaches to parallel runways $17 \mathrm{C}$ and $17 \mathrm{R}$ respectively from opposite sides. The weight classes of both aircraft are "large", so wake turbulence separation rules do not apply. The runways are separated by only $1300 \mathrm{ft}$. The squares are waypoints of the NIRs, which end at the runway thresholds near the arrows for the flight directions. The solid and dashed lines are the actual trajectories for aircraft $\mathrm{ABC} 1213$ and $\mathrm{ABC} 2371$ respectively. The dark solid lines are predicted trajectories starting from the current positions to the first predicted violation of the safety conformance alert threshold of 0.75 for aircraft approaching parallel runways less than $2500 \mathrm{ft}$ apart but not yet established on their final approach courses. The circles are of diameter $2.25 \mathrm{NM}$ ( $75 \%$ of $3 \mathrm{NM})$. Note that, once the aircraft are established on their final approach courses, there will not be alerts even if the succeeding aircraft moves forward to a nearly sideby-side position with zero vertical separation. In this case, aircraft $\mathrm{ABC} 2371$ did not move forward as indicated by the stars, which are one minute apart. At the current position where the trajectories were predicted, they leveled temporarily at $3000 \mathrm{ft}$. The predictions disappeared when aircraft $\mathrm{ABC} 237$ continued the base turn. This alert may again be on the gray area between valid and nuisance alerts. A small number of this kind of alert may be acceptible to the controller. It also shows that the aircraft made the turn to maintian proper reduced separation for visual approaches. STARS CA did not alert for this pair. The inset provides a close-up view of the

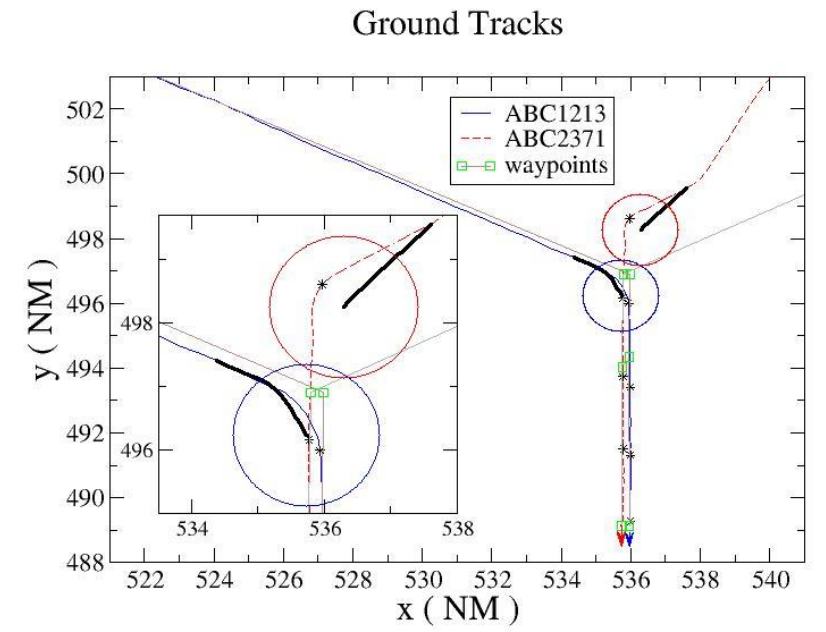

Figure 17. Ground tracks for an arrival pair making visual final approaches to two parallel runways less than $2500 \mathrm{ft}$ apart from opposite sides with track crossing. encounter.

We notice from the predicted trajectories that the headings of aircraft ABC128 in Fig. 15, ABC2516 in Fig.16, and ABC2371 in Fig. 17 all lag behind their actual headings at their starting positions of the predicted conflicts. This lagging is a result of the filtering algorithms used in the NASA recorded data. Without the heading lag, the three alerts may not appear and the safety alert thresholds may be increased further to provide larger alert lead time without proliferating nuisance alerts.

\section{Conclusion}

A set of safety alert thresholds, which allow safety alerts to be provided to the controllers for aircraft conducting visual approaches to a single runway or multiple parallel runways, has been proposed based on input from Subject Matter Experts as well as visual approach procedures and common practices. The goal is to maximize the thresholds within the guidance of the procedures and common practices and to minimize the number of nuisance alerts and total number of alerts with the support of flight intent information. Tests have been performed using a recently developed prototype tactical separation assurance system for terminal airspace, called Terminal Tactical Separation-Assured Flight Environment (T-TSAFE). The input was a full day of air traffic data from Dallas/Fort Worth (DFW) TRACON with most arriving flights conducting visual approaches. The results compare favorably with those of the Conflict Alert (CA) functionality of the Standard Terminal Automation Replacement System (STARS).

When fast-time simulation experiment was performed using T-TSAFE with all arriving flights assumed to conduct instrument approaches, the expected large number of separation alerts were observed. When compared with STARS CA, the conflict pairs common to both T-TSAFE and STARS CA was only $21 \%$ of the total STARS CA alerts. As a result, the nuisance-alert rate for STARS CA was estimated to be about $70 \%$, which is comparable to a similar previous 
estimation of $80 \%$ nuisance-alert rate for CARTS (Common Automated Radar Terminal System) CA. The Mode-C Intruder (MCI) alerts were also reduced by $76 \%$ as compared to STARS CA.

Examination of the common conflict pairs between T-TSAFE and STARS CA shows that they are valid separation conflicts with good alert lead times for T-TSAFE. However, many of them would still be considered nuisance alerts if the aircraft were conducting visual approaches. This was confirmed by another visual approach fast-time simulation T-TSAFE experiment, in which all DFW arriving flights were assumed to be on visual approaches and our proposed safety alert thresholds were used and tested. The result of the experiment showed that the number of non-MCI alerts was less by $92 \%$ as compared to the number when all DFW arrivals were assumed to be conducting instrument approaches. The common conflict pairs involving aircraft conducting visual approaches to parallel runways were reduced by $93 \%$ as well. Thus, the flight intent information and the safety alert thresholds are effective in reducing nuisance alerts.

T-TSAFE can thus provide separation and safety conflict alerts seamlessly in the real-world environment of mixed terminal operations with arriving flights of both visual and instrument approaches. Compared with STARS CA, TTSAFE has fewer false alerts, larger alert lead time, and larger alert thresholds. While the nuisance alerts are reduced significantly with the safety alert thresholds, further work is needed to study if the alert lead time for safety alerts is sufficient.

\section{References}

1 "Common ARTS Computer Program Functional Specifications (CPFS), Conflict Alert”, Federal Aviation Administration, NAS-MD-632, Washington D.C., Apr. 2007.

2 “Standard Terminal Automation Replacement System (STARS), Adaptation Data Maintenance Manual, Revision 20", Raytheon, Prepared for Federal Aviation Administration, NAS-MD-4428, Washington D.C., Jan. 2014.

3“Air Traffic Control", Federal Aviation Administration, Order JO 7110.65V, Washington, DC. Feb. 2014. http://www.faa.gov/documentLibrary/media/Order/JO_7110.65V.pdf [retrieved 1 Sep. 2014].

${ }^{4}$ Tang, H., Robinson, J.E., and Denery, D.G., "Tactical Conflict Detection in Terminal Airspace”, AIAA, Journal of Guidance, Control, and Dynamics, Vol.34, No.2, 2011, pp 403-413.

${ }^{5}$ Tang, H., "Severity-Based Tactical Conflict Detection in Terminal Airspace", AIAA Paper 2012-5646, September, 2012.

${ }^{6}$ Tang, H., "A Tactical Separation Assurance System for Terminal Airspace”, AIAA Paper 2014-2021, June, 2014.

${ }^{7}$ Friedman-Berg F, Allendoerfer K., and Shantanu Pai, "Nuisance Alerts in Operational ATC Environments: Classification and Frequencies", Proceedings of the human factors and ergonomics society, 2008.

8 "Air Traffic Quality Assurance", Federal Aviation Administration, Order JO 7210.56C, Washington, DC. July. 2009. http://www.faa.gov/documentLibrary/media/Order/ATQ.pdf [retrieved 1 Aug. 2012].

${ }^{9}$ Swenson, H. N., Hoang, T., Engelland, S., Vincent, D., Sanders, T., Sanford, B., and Heere, K., "Design and Operational Evaluation of the Traffic Management Advisor at the Fort Worth Air Route Traffic Control Center," 1st USA/Europe Air Traffic Management R\&D Seminar, Saclay, France, Federal Aviation Admin. And EUROCONTROL, Paper 012, June 1997.

10 "Traffic Flow Management in the National Airspace System", Federal Aviation Administration, Washington, DC. Oct. 2009. http://www.fly.faa.gov/Products/Training/Traffic_Management_for_Pilots/TFM_in_the_NAS_Booklet_ca10.pdf [retrieved 1 Apr. 2014]. 